\title{
Prostaglandin E1 attenuates post-cardiac arrest myocardial dysfunction through inhibition of mitochondria-mediated cardiomyocyte apoptosis
}

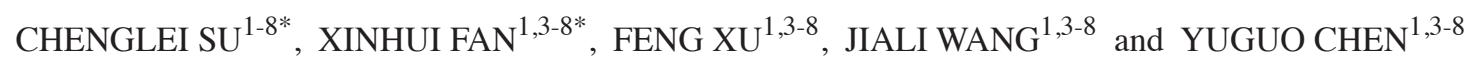

${ }^{1}$ Department of Emergency Medicine, Qilu Hospital, Cheeloo College of Medicine, Shandong University, Jinan, Shandong 250012; ${ }^{2}$ Department of Emergency, The Affiliated Hospital of Xuzhou Medical University, Xuzhou,

Jiangsu 221000; ${ }^{3}$ Shandong Provincial Clinical Research Center for Emergency and Critical Care Medicine, Institute of Emergency and Critical Care Medicine of Shandong University, Qilu Hospital, Cheeloo College of Medicine, Shandong University; ${ }^{4}$ Key Laboratory of Emergency and Critical Care Medicine of Shandong Province;

${ }^{5}$ Key Laboratory of Cardiopulmonary-Cerebral Resuscitation Research of Shandong Province;

${ }^{6}$ Shandong Provincial Engineering Laboratory for Emergency and Critical Care Medicine;

${ }^{7}$ The Key Laboratory of Cardiovascular Remodeling and Function Research, Chinese Ministry of Education,

Chinese Ministry of Health and Chinese Academy of Medical Sciences, Qilu Hospital, Cheeloo College of Medicine, Shandong University; ${ }^{8}$ The State and Shandong Province Joint Key Laboratory of Translational Cardiovascular Medicine, Qilu Hospital, Cheeloo College of Medicine, Shandong University, Jinan, Shandong 250012, P.R. China

Received June 11, 2020; Accepted October 19, 2020

DOI: $10.3892 / \mathrm{mmr} .2020 .11749$

\begin{abstract}
Post-cardiac arrest myocardial dysfunction (PAMD) is a leading cause of death in patients undergoing resuscitation patients following cardiac arrest (CA). Although prostaglandin E1 (PGE1) is a clinical drug used to mitigate ischemia injury, its effect on PAMD remains unknown. In the present study, the protective effects of PGE1 on PAMD were evaluated in a rat model of $\mathrm{CA}$ and in a hypoxia-reoxygenation $(\mathrm{H} / \mathrm{R})$ in vitro model. Rats were randomly assigned to $\mathrm{CA}, \mathrm{CA}+\mathrm{PGE} 1$ or sham groups. Asphyxia for 8 min followed by cardiopulmonary resuscitation were performed in the CA and CA+PGE1 groups. PGE1 was intravenously administered at the onset of return of spontaneous circulation (ROSC). PGE1 treatment significantly increased the ejection fraction and cardiac output within $4 \mathrm{~h}$ following ROSC and improved the survival rate, compared with the CA group. Moreover, PGE1
\end{abstract}

Correspondence to: Dr Yuguo Chen or Dr Jiali Wang, Department of Emergency Medicine, Qilu Hospital, Cheeloo College of Medicine, Shandong University, 107 Wenhuaxi Road, Jinan, Shandong 250012, P.R. China

E-mail: chen919085@sdu.edu.cn

E-mail: wangjiali_2000@126.com

*Contributed equally

Key words: prostaglandin E1, cardiac arrest, post-cardiac arrest myocardial dysfunction, mitochondrial permeability transition pore, GSK3 $\beta$ inactivated GSK3 $\beta$, prevented mitochondrial permeability transition pore (mPTP) opening, while reducing cytochrome $c$ and cleaved caspase-3 expression, as well as cardiomyocyte apoptosis in the rat model. To examine the underlying mechanism, H/R H9c2 cells were treated with PGE1 at the start of reoxygenation. The changes in GSK $3 \beta$ activity, $\mathrm{mPTP}$ opening, cytochrome $c$ and cleaved caspase-3 expression, and apoptosis of $\mathrm{H} 9 \mathrm{c} 2$ cells were consistent with those noted in vivo. The results indicated that PGE1 attenuated PAMD by inhibiting mitochondria-mediated cardiomyocyte apoptosis.

\section{Introduction}

Cardiac arrest (CA) poses a significant public health burden. Although modern cardiopulmonary resuscitation (CPR) improves the return of spontaneous circulation (ROSC) rate in patients who have suffered from CA, the survival to hospital discharge rate remains poor $(1,2)$. Significant left ventricular systolic and diastolic dysfunction early after ROSC is termed post-cardiac arrest myocardial dysfunction (PAMD) and is a common symptom noted in these patients (3). Although PAMD is temporary following resuscitation, approximately two-thirds of the patients who achieve ROSC do not survive due to PAMD within the first $72 \mathrm{~h}$ (4-6). Currently, pharmacological treatments that attenuate PAMD are not available $(6,7)$.

Mitochondria-mediated cardiomyocyte apoptosis is one of the primary mechanisms underlying PAMD due to CA $(6,8,9)$. Global myocardial ischemia associated with CA induces mitochondrial permeability transition pore (mPTP) opening, which results in cytochrome $c$ leakage from the mitochondrial matrix to the cytoplasm, leading to caspase-3 activation and induction of apoptosis (10-12). Inhibition of mPTP opening 
and apoptosis induction may represent a potential therapeutic approach for PAMD $(13,14)$.

Prostaglandin E1 (PGE1) is an essential member of the prostaglandin family that demonstrates several physiological and pharmacological activities. PGE1 is widely used in the clinic for the treatment of ischemic injury (15-17). PGE1 decreases mPTP opening and apoptosis in animal models of myocardial infarction and coronary microembolization (17-19). However, protective effects of PGE1 on PAMD have not been reported to date.

The present study evaluated whether PGE1 treatment could attenuate PAMD and improve survival rates in a rat model of CA. In addition, whether PGE1 might exert cardioprotective effects by inhibiting mitochondria-mediated cardiomyocyte apoptosis was also assessed.

\section{Materials and methods}

Animals. Animal experiments were conducted following the guide for the Care and Use of Laboratory Animals of the National Institutes of Health. The present study was approved by the Animal Use and Care Committee of Shandong University. Male Wistar rats weighing 380-430 g, aged 14 weeks were purchased from the Department of Experimental Animals of Shandong University. The rats were housed in independent ventilation cages at an ambient temperature of $23 \pm 1^{\circ} \mathrm{C}$ and a humidity of $55 \pm 5 \%$ under a 12 -h light/dark cycle. They were allowed access to food and tap water ad libitum.

Asphyxia-induced CA model establishment and animal treatments. A previously published rat model of CA was used in the present study. Briefly, rats were anesthetized with $5 \%$ isoflurane in room air ( $21 \%$ oxygen) in a plastic induction box. The trachea was orally intubated once the rats were fully anesthetized (no response to pain absence of corneal reflex). The rats were mechanically ventilated and maintained under anesthesia with $2 \%$ isoflurane. A PE-50 catheter was advanced through the left femoral artery into the aorta for measurement of the mean aortic pressure (MAP). A microcatheter was inserted into the left femoral vein for drug administration. A conventional lead II electrocardiogram was continuously recorded. Pancuronium bromide $(2 \mathrm{mg} / \mathrm{kg}$ ) was intravenously administered for complete muscle relaxation. Following pancuronium bromide administration, the rats were administered $0.5 \%$ isoflurane in room air (21\% oxygen) for $5 \mathrm{~min}$.

The rats were randomly allocated to three groups as follows (Fig. 1): i) In the CA+PGE1 group, the rats underwent 8 min asphyxia followed by CPR, and $1 \mu \mathrm{g} / \mathrm{kg}$ Lipo-PGE1 (Qilu Pharmaceutical Co., Ltd.) was intravenously administered at the onset of $\operatorname{ROSC}(19,20)$; ii) in the CA group, the rats underwent 8 min of asphyxia and CPR; and iii) in the sham group, the rats underwent the same surgical procedure without PGE1 or asphyxia/CPR. The rat model of asphyxia-induced CA was established as described previously $(21,22)$. Following pancuronium-bromide administration, the rats were administered $0.5 \%$ isoflurane in room air (21\% oxygen) for $5 \mathrm{~min}$. CA was induced by asphyxia, which occurred after turning off the ventilator and clamping the endotracheal tube. The hypotension and heart rate dropped quickly. CA was defined as a MAP of $<20 \mathrm{mmHg}$. Following $8 \mathrm{~min}$ of asphyxia, chest compression $(23,24)$ was initiated at a rate of 200 beats $/ \mathrm{min}$ with a pneumatically driven mechanical chest compressor (Fig. S1). The depth of compressions $(1-1.5 \mathrm{~cm})$ was adjusted to maintain the MAP > $25 \mathrm{mmHg}$ during resuscitation. Mechanical ventilation with $100 \%$ oxygen and a bolus injection of $10 \mu \mathrm{g} / \mathrm{kg}$ epinephrine via a venous line were initiated $30 \mathrm{sec}$ prior to chest compression. Chest compressions were continued and epinephrine was injected every 5 min until ROSC was achieved. ROSC was defined as the return of supraventricular rhythm with an increase in MAP $>50 \mathrm{mmHg}$ for $5 \mathrm{~min}$. Only rats that achieved ROSC within 10 min were used for further studies (25-27). This criterion was to avoid non-homogeneous CA periods leading to pathophysiological differences and subsequently to the need for larger numbers of animals. In this CA model, most of rats could get back to supraventricular rhythm within 5 min after performing CPR and maintain MAP more than $50 \mathrm{mmHg}$. At $4 \mathrm{~h}$ post-ROSC, 21 rats (n=7/group) were euthanized with Euthasol $(390 \mathrm{mg} / \mathrm{kg}$ pentobarbital sodium and $50 \mathrm{mg} / \mathrm{kg}$ phenytoin sodium; intraperitoneal injection) (28). Death was confirmed by the cessation of the heartbeat and respiration. The remaining 30 rats ( $n=10 /$ group) were monitored for 72-h survival analysis.

Cardiac function monitoring. Cardiac function was measured using an ultra-high frequency ultrasound system for small animals (Vevo 2100; VisualSonics, Inc.) at baseline and 1, 2, 3 and $4 \mathrm{~h}$ post-ROSC. Left ventricular end-diastolic diameter (LVEDD), left ventricular end-systolic diameter (LVESD), left ventricular end-diastolic volume (LVEDV), left ventricular end-systolic volume (LVESV) and heart rate (HR) were recorded from M-mode images. Ejection fraction (EF) was calculated as EF=[(LVEDV-LVESV)/LVEDV] $\mathrm{x} 100$. Cardiac output was calculated as $\mathrm{CO}=($ LVEDV-LVESV $) \mathrm{x}$ HR. All measurements were performed and reviewed by two investigators blinded to group assignment.

Survival analysis. For the 72-h survival study, all catheters were removed and wounds were surgically closed at $4 \mathrm{~h}$ following ROSC. Rats received a subcutaneous injection of $1 \mathrm{mg} / \mathrm{kg}$ buprenorphine to relieve pain when they were returned to their cages. Rats were observed every $2 \mathrm{~h}$ within first $24 \mathrm{~h}$ after ROSC. Then rats were observed at 48 and $72 \mathrm{~h}$. During the 72-h observation period, gasping or respiratory rate under 5 breaths/min required immediate euthanasia (29). At 72 h, all surviving rats were re-anesthetized with $5 \%$ isoflurane and had a vein catheter placed for blood sampling. At the end of the experiment rats were euthanized.

Hypoxia-reoxygenation $(H / R)$ cell model establishment. The $\mathrm{H} 9 \mathrm{c} 2$ rat cardiomyoblast cell line was obtained from the Cell Bank of the Chinese Academy of Sciences. H9c2 cells were grown in high-glucose DMEM (Gibco; Thermo Fisher Scientific, Inc.) supplemented with $10 \%$ fetal bovine serum (Gibco; Thermo Fisher Scientific, Inc.) and 1\% penicillin/streptomycin at $37^{\circ} \mathrm{C}$ in a humidified atmosphere of $5 \% \mathrm{CO}_{2}$.

In the present study, $\mathrm{H} 9 \mathrm{C} 2$ cells were digested and divided into three groups. The control group was incubated in normal culture medium. The H/R group was treated as described previously $(30,31)$. Briefly, H9c2 cells in glucose-free DMEM (Gibco; Thermo Fisher Scientific, Inc.) were exposed to $1 \% \mathrm{O}_{2}$, 
* Cardiac function monitoring

\# TUNEL/mPTP opening/Western blot analysis

$\S$ Survival analysis

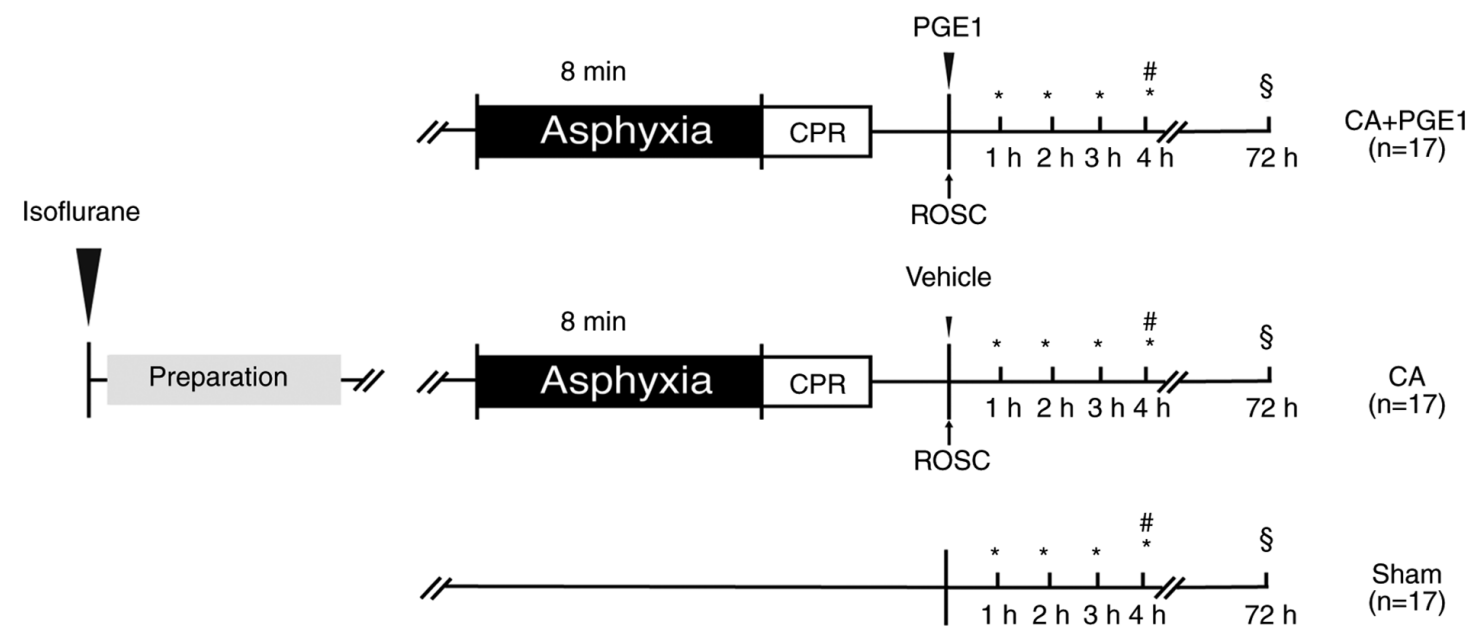

Figure 1. Experimental design of the in vivo study. CA, cardiac arrest; CPR, cardiopulmonary resuscitation; ROSC, return of spontaneous circulation; PGE1, prostaglandin E1; mPTP, mitochondrial permeability transition pore.

$94 \% \mathrm{~N}_{2}$ and $5 \% \mathrm{CO}_{2}$ in an anaerobic chamber (Don Whitley Scientific, Ltd.) for $12 \mathrm{~h}$ to mimic ischemia. Subsequently, the medium was replaced with high-glucose DMEM and the cells were transferred to a regular incubator at $37^{\circ} \mathrm{C}$ with $5 \% \mathrm{CO}_{2}$ for $12 \mathrm{~h}$ to mimic reperfusion. To study the effects of PGE1 on $\mathrm{H} / \mathrm{R}$ model, the $\mathrm{H} / \mathrm{R}+\mathrm{PGE} 1$ group was treated with $0.5 \mu \mathrm{M}$ PGE1 at the start of reoxygenation.

TUNEL assay. Cardiomyocyte apoptosis was detected in rats using a TUNEL assay using with the in situ Apoptosis Detection Kit (EMD Millipore) according to the manufacturer's instructions. Briefly, the heart tissue was separated and fixed in $4 \%$ paraformaldehyde overnight at room temperature. Fixed heart tissue was embedded in paraffin and cut transversely into $5-\mu \mathrm{m}$ sections. TUNEL reaction mixture $(100 \mu \mathrm{l})$ was added to cover the tissue section and incubated in a humidified chamber for $1 \mathrm{~h}$ at $37^{\circ} \mathrm{C}$. The nuclei were counterstained with $0.5 \%$ methyl green for $5 \mathrm{~min}$ at room temperature. The tissue sections were washed with xylene (three washes; each wash, $2 \mathrm{~min}$ ) and covered with coverslips using neutral balsam mounting medium. The sections were examined under a BX41 light microscope (Olympus Corporation) and images of selected areas were captured.

H9c2 cell apoptosis was detected using a TUNEL assay with an Apoptosis Assay Kit (Roche Diagnostics), according to the manufacturer's instructions. Briefly, the H9c2 cells were fixed in $4 \%$ paraformaldehyde for $1 \mathrm{~h}$ at room temperature. The samples were incubated with the TUNEL reaction mixture $(50 \mu \mathrm{l})$ at $37^{\circ} \mathrm{C}$ for $60 \mathrm{~min}$. The nuclei were counterstained with DAPI for $5 \mathrm{~min}$ at room temperature. The samples were washed in PBS (three washes; each wash, $5 \mathrm{~min}$ ) and coverslipped in fluorescence mounting medium. The cells were visualized under an IX73 fluorescence microscope (Olympus Corporation) using excitation and emission wavelengths of 540 and $580 \mathrm{~nm}$, respectively. The cells exhibiting red fluorescence were defined as TUNEL-positive apoptotic cells.
The levels of apoptosis in tissue and H9c2 cells were calculated by counting the TUNEL-positive cardiomyocyte nuclei in three randomly selected fields in each slide (magnification, $\mathrm{x} 400$ ). The levels were reported as a percentage of total cardiomyocyte nuclei.

Measurement of MPTP opening. The mitochondria derived from rat myocardium and/or from $\mathrm{H} 9 \mathrm{c} 2$ cells were isolated by differential centrifugation using a Tissue/Cell Mitochondria Isolation Kit (Beyotime Institute of Biotechnology) according to the manufacturer's instructions. Fresh mitochondria were used for the measurement of mPTP opening, while the mitochondria-free cytoplasmic protein extract was used for the measurement of cytochrome $c$ expression.

mPTP opening was measured using a Purified Mitochondrial Membrane Pore Channel Colorimetric Assay kit (Genmed). mPTP opening was induced by $200 \mu \mathrm{M} \mathrm{CaCl}_{2}$ and presented as mitochondrial swelling, which results in a reduction of the absorbance at $520 \mathrm{~nm}\left(\mathrm{~A}_{520}\right)$. The changes in $\mathrm{A}_{520}$ at various time points were measured for each sample. The value at $-1 \mathrm{~min}$ was normalized to the value at $10 \mathrm{~min}$ and was used for statistical analysis.

An additional immunofluorescence method was used to measure mPTP opening in H9c2 cells. Briefly, cells were washed with PBS and subsequently stained with $1 \mu \mathrm{mol} / 1$ calcein-AM (Invitrogen; Thermo Fisher Scientific, Inc.) in the presence of $8 \mathrm{mmol} / 1 \mathrm{CoCl}_{2}$ (Sigma-Aldrich; Merck KGaA) at room temperature for $20 \mathrm{~min}$ in the dark. $\mathrm{CoCl}_{2}$ was added to quench the cytoplasmic signal so that only fluorescence in the mitochondria was captured. A change in fluorescence intensity corresponded to the level of mPTP opening.

Western blot analysis. Total protein was extracted from heart tissues and H9c2 cells using 1X RIPA buffer (Sigma-Aldrich; Merck KGaA) with a Protease Inhibitor Cocktail (Sigma-Aldrich; Merck KGaA). The protein concentration was determined using the Pierce BCA Protein Assay (Pierce; Thermo Fisher 
Table I. Baseline characteristics of rats and resuscitation characteristics.

\begin{tabular}{lccc}
\hline Variable & CA & CA+PGE1 & Sham \\
\hline Body weight, $(\mathrm{g})$ & $400.7 \pm 17.8$ & $411.4 \pm 14.7$ & $413.5 \pm 10.1$ \\
Heart rate before asphyxia, bpm & $357.2 \pm 33.4$ & $363.5 \pm 45.6$ & $340.8 \pm 29.9$ \\
MAP before asphyxia, $\mathrm{mmHg}$ & $133.7 \pm 15.6$ & $131.8 \pm 18.9$ & $137.3 \pm 10.1$ \\
MAP at 1 h after ROSC, $\mathrm{mmHg}$ & $96.2 \pm 15.2$ & $94 \pm 17.83$ & - \\
Cardiac arrest time, sec & $274.9 \pm 37.2$ & $271.1 \pm 29.8$ & - \\
CPR duration, sec & $43.0 \pm 12.1$ & $43.7 \pm 16.5$ & - \\
Adrenaline dose, $\mu \mathrm{g}$ & $4.1 \pm 0.2$ & $4.1 \pm 0.2$ & - \\
\hline
\end{tabular}

$\mathrm{n}=17$ in each group. $\mathrm{CA}$, cardiac arrest; PGE1, prostaglandin E1; MAP, mean aortic pressure; CPR, cardiopulmonary resuscitation; bpm, beats per minute; $\mathrm{mmHg}$, millimeters of mercury.

Scientific, Inc.). Total protein was used to measure the expression levels of GSK3 $\beta$ and caspase-3. Cytoplasmic protein without mitochondria was used to measure cytochrome $c$ expression. The samples with equal amounts of protein $(50 \mu \mathrm{g})$ were loaded onto $10 \%$ sodium dodecyl sulfate-polyacrylamide gels, subjected to electrophoresis and subsequently transferred onto $0.22-\mu \mathrm{M}$ PVDF membranes (EMD Millipore). Following blocking with $5 \%$ nonfat milk for $1 \mathrm{~h}$ at room temperature, the membranes were incubated with primary antibodies overnight at $4^{\circ} \mathrm{C}$ : Specific for total GSK3 $\beta$ (1:1,000; cat. no. 12456 ; Cell Signaling Technology, Inc.), phosphorylated (p)-GSK3 $\beta$ (1:1,000; Ser9; cat. no. 55558; Cell Signaling Technology, Inc.), caspase-3 (1:1,000; cat. no. 9662; Cell Signaling Technology, Inc.), cleaved-caspase-3 (1:1,000; cat. no. 9664; Cell Signaling Technology, Inc.), cytochrome $c$ (1:1,000; cat. no. 11940; Cell Signaling Technology, Inc.). $\beta$-actin (1:1,000; cat. no. 60008-1; ProteinTech Group, Inc.) was used to detect reference protein expression. After incubation with an HRP-conjugated anti-rabbit (1:20,000; cat. no. ab205718; Abcam) or anti-mouse secondary antibody (1:20,000; cat. no. ab205719; Abcam) for $1 \mathrm{~h}$ at room temperature, the labeled proteins were visualized using the Pierce ECL Western Blotting Substrate (Pierce; Thermo Fisher Scientific, Inc.). Signals from immunoblots were scanned using Amersham Imager 600 imagers (GE Healthcare). The relative band intensities were quantified using the ImageJ software (version 1.52a; National Institutes of Health).

Statistical analysis. All data are presented as the mean \pm SD of three independent experiments. Multi-group comparisons were performed using one-way ANOVA followed by Tukey's post hoc test. Student's t-test was used to compare two groups. The comparisons between time-based measurements within each group were performed using repeated-measures ANOVA. Survival was analyzed using Kaplan-Meier curves and log-rank tests. Two-sided $\mathrm{P}<0.05$ was considered to indicate a statistically significant difference. Statistical analysis was carried out using GraphPad Prism 7.0 (GraphPad Software, Inc.).

\section{Results}

Baseline characteristics of rats and resuscitation characteristics. In total, 58 rats were used in the present study, of which 51 rats achieved ROSC within $10 \mathrm{~min}$ and were included in further studies. Two rats died for the anesthesia induction before CA, and 5 rats were euthanized because that they did not get back to supraventricular rhythm after 5 min CPR or could not maintain MAP more than $50 \mathrm{mmHg}$ for $5 \mathrm{~min}$ within $10 \mathrm{~min}$. The mortality rate during model establishment in this study was consistent with the previous report (27).

No significant differences were noted in the parameters body weight, baseline MAP and HR between the treatment groups (Table I). No significant differences were noted in the duration of CA, epinephrine dose, duration of CPR, MAP and $\mathrm{HR}$ at $1 \mathrm{~h}$ following ROSC between the CA and the CA+PGE1 groups (Table I).

PGE1 ameliorates cardiac dysfunction. $\mathrm{CO}$ and EF were measured in the three treatment groups at baseline and at 1,2, 3 and 4 h following ROSC (Fig. 2). No significant differences were noted in baseline cardiac function between the groups. $\mathrm{CO}$ and EF were significantly decreased within $4 \mathrm{~h}$ following ROSC in the CA group compared with the sham group. However, CO was significantly increased following PGE1 treatment, compared with the CA group $(\mathrm{P}<0.05)$. A highly significant difference was also noted at $4 \mathrm{~h}$ following ROSC when comparing the mean EF values between the CA and $\mathrm{CA}+\mathrm{PGE} 1$ groups $(65.8 \pm 16.2$ vs. $81.2 \pm 9.91 \%$; $\mathrm{P}<0.01)$.

PGE1 improves the survival rate. The survival rates were monitored for $72 \mathrm{~h}$. Kaplan-Meier survival curves indicated a rapid decline in the survival rate of rats in the CA group within $24 \mathrm{~h}$ following ROSC (Fig. 3). The survival rate in the $\mathrm{CA}+\mathrm{PGE1}$ group was significantly higher than that in the CA group $(\mathrm{P}<0.05$; Fig. 3$)$.

PGE1 suppresses cardiomyocyte apoptosis. Myocardial apoptosis was detected using TUNEL staining. In vivo, the levels of apoptosis in the CA group were significantly increased compared with those in the sham group $(12.11 \pm 4.37$ vs. $48.33 \pm 19.07 \%, \mathrm{P}<0.05$, Fig. 4A). In contrast to these findings, the levels of cardiac apoptosis decreased following treatment with PGE1, compared with the CA group (31.02 \pm 13.51 vs. $48.33 \pm 19.07$, respectively; $\mathrm{P}<0.05$; Fig. 4A). In vitro, the fraction of apoptotic $\mathrm{H} 9 \mathrm{c} 2$ cells was significantly higher in the in $\mathrm{H} / \mathrm{R}$ group than that in the control group $(52.13 \pm 7.86$ vs. $6.38 \pm 2.02 \%$, respectively; $\mathrm{P}<0.01$; Fig. $4 \mathrm{~B})$. 

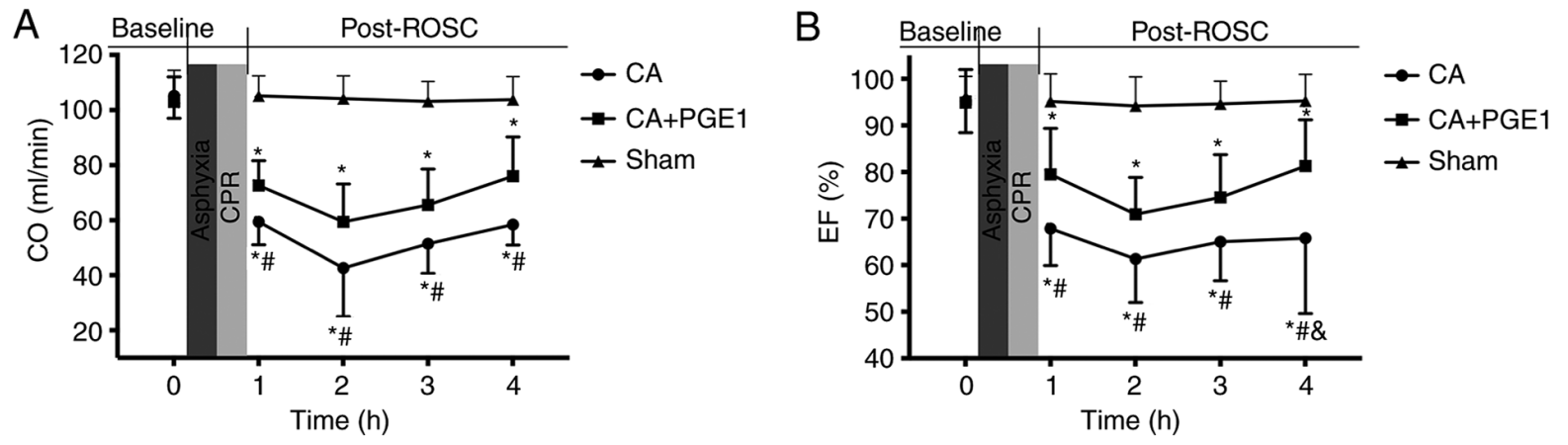

Figure 2. Changes in cardiac function. (A) $\mathrm{CO}$ and (B) EF in the sham, CA and CA+PGE1 groups from baseline to $4 \mathrm{~h}$ following ROSC. $\mathrm{n}=17$ in each group. ${ }^{*} \mathrm{P}<0.05$ vs. sham group; ${ }^{\#} \mathrm{P}<0.05$ vs. $\mathrm{CA}+\mathrm{PGE} 1$ group; ${ }^{\&} \mathrm{P}<0.01$ vs. CA+PGE1 group. CA, cardiac arrest; CO, cardiac output; ROSC, return of spontaneous circulation; EF, ejection fraction; PGE1, prostaglandin E1.

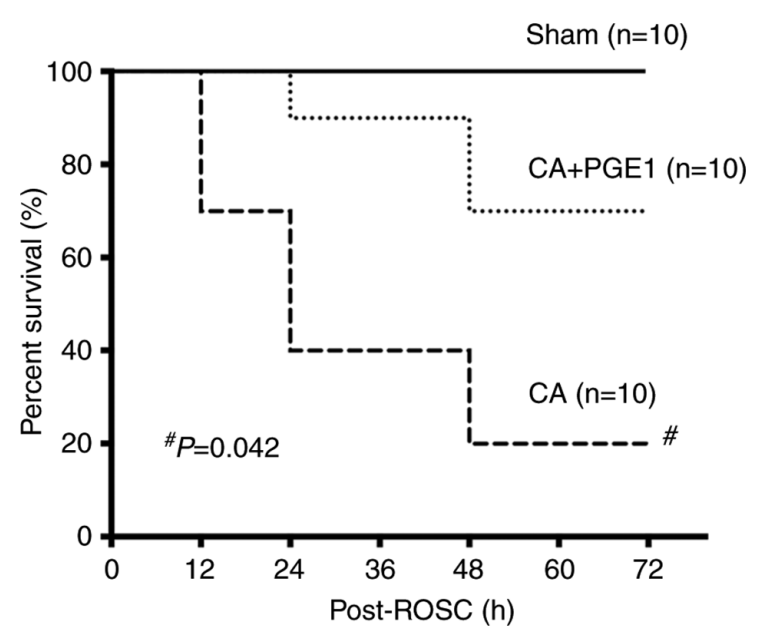

Figure 3. Survival rate during the first $72 \mathrm{~h}$ following ROSC. ${ }^{\#} \mathrm{P}<0.05$ vs. $\mathrm{CA}+\mathrm{PGE} 1$ group. $\mathrm{CA}$, cardiac arrest; ROSC, return of spontaneous circulation; PGE1, prostaglandin E1.

The fraction of apoptotic H9c2 cells in the H/R+PGE1 group significantly decreased, compared with the H/R group (31.20 \pm 12.62 vs. $52.13 \pm 7.86 \%$, respectively; $\mathrm{P}<0.01$, Fig. 4B).

PGE1 blocks mPTP opening. The mitochondrial swelling assay results demonstrated that the change of $\mathrm{OD}$ value $\mathrm{A}_{520}$ in the CA group was lower compared with the sham group $(0.61 \pm 0.11$ vs. $0.82 \pm 0.13$, respectively; $\mathrm{P}<0.05$; Fig. $5 \mathrm{~A}$ and $\mathrm{B})$, which indicated the level of mPTP opening was increased in the CA group. PGE1 treatment reduced mPTP opening, compared with the CA group $(0.76 \pm 0.11$ vs. $0.61 \pm 0.12$, respectively; $\mathrm{P}<0.05$; Fig. 5A and B). Moreover, mPTP opening was decreased by PGE1 treatment in vitro, compared with the $\mathrm{H} / \mathrm{R}$ group $(0.77 \pm 0.13$ vs. $0.58 \pm 0.10$, respectively; $\mathrm{P}<0.05$; Fig. 5C and D). Consistent with the mitochondrial swelling assay results, the relative fluorescence intensity of calcein-AM was significantly increased in the $\mathrm{H} / \mathrm{R}+\mathrm{PGE} 1$ group, compared with the H/R group $(0.72 \pm 0.24$ vs. $0.41 \pm 0.12$, respectively; $\mathrm{P}<0.01$; Fig. 5E-H).

PGE1 regulates GSK3 $\beta$, cleaved caspase-3 and cytochrome c expression. GSK $3 \beta$ protein phosphorylation (Ser9) was also analyzed. In vivo, GSK $3 \beta$ phosphorylation decreased in the CA group compared with the sham group $(\mathrm{P}<0.01$; Fig. $6 \mathrm{~A})$.
GSK $3 \beta$ phosphorylation was higher in the CA+PGE1 group than in the CA group $(\mathrm{P}<0.01$; Fig. $6 \mathrm{~A})$. In vitro, GSK $3 \beta$ phosphorylation was decreased in the $\mathrm{H} / \mathrm{R}$ group $(\mathrm{P}<0.01)$, compared with the control group. However, it was partly restored following PGE1 treatment $(\mathrm{P}<0.01$; Fig. 6B).

The cytoplasmic protein levels of cleaved caspase-3 and cytochrome $c$ were significantly increased in the CA group in vivo, compared with the sham group ( $\mathrm{P}<0.01$; Fig. 6A). PGE1 treatment partially suppressed these effects $(\mathrm{P}<0.01$; Fig. 6B). The results obtained in vitro were consistent with the in vivo data. Indeed, PGE1 treatment inhibited the increase in the expression levels of cleaved caspase-3 and cytochrome $c$ induced by $H / R(P<0.01$; Fig. $6 B)$.

\section{Discussion}

PAMD is a common condition that can reduce survival in resuscitated patients following CA. No effective treatment strategies have been reported for PAMD $(6,32)$. In the present study, it was demonstrated that PGE1 treatment improved cardiac function and survival outcome following ROSC in a rat model of asphyxia-induced CA. Moreover, in vitro experiments demonstrated that inhibition of mitochondria-mediated cardiomyocyte apoptosis was involved in the mechanism underlying the protective effect of PGE1.

The rat model of asphyxia-induced CA used in the present study is commonly used (33). The EF and CO parameters were measured in the experimental animals, which confirmed a significant impairment of cardiac function following CA. It is interesting to note that PGE1 treatment attenuated cardiac dysfunction at $1 \mathrm{~h}$ following ROSC. Furthermore, PGE1 significantly improved EF at $4 \mathrm{~h}$ following ROSC. These results indicated that PGE1 exhibited protective effects against PAMD.

The underlying mechanism was investigated in the in vivo and in vitro models. The primary causes of PAMD are myocardial apoptosis and stunning following ischemiareperfusion injury $(6,34)$. Inhibition of apoptosis can effectively alleviate organ disorders (35). Gu et al (36) reported that inhibition of cardiomyocyte apoptosis could improve cardiac function following CA. In the present study, the induction of cardiomyocyte apoptosis was investigated following CA. The results indicated that PGE1 treatment significantly ameliorated cardiomyocyte apoptosis following CA. 
A
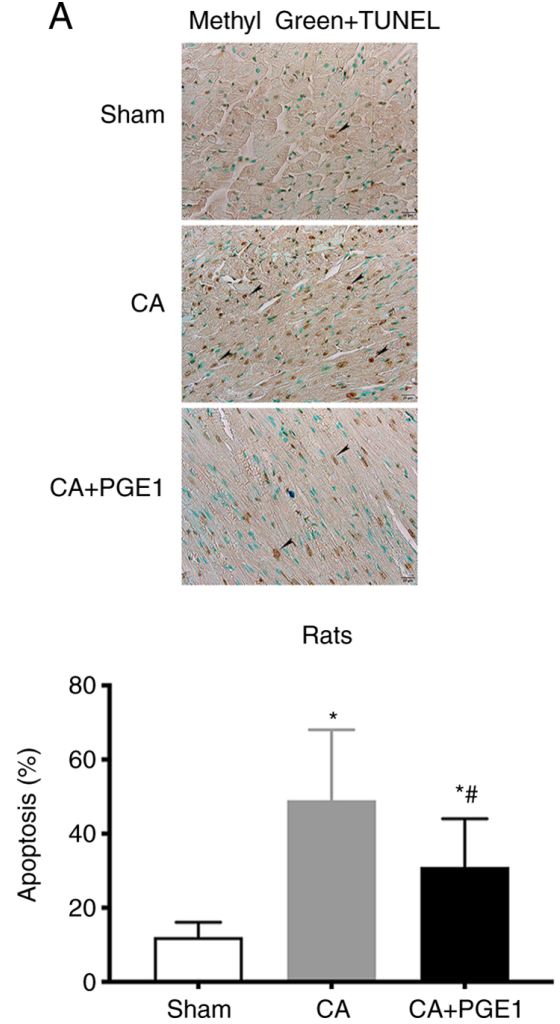

B

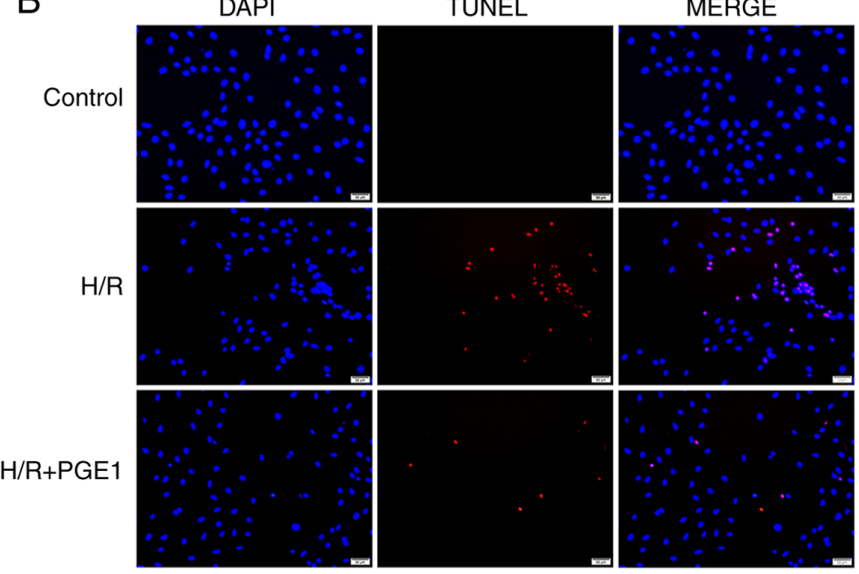

$\mathrm{H} 9 \mathrm{c} 2$

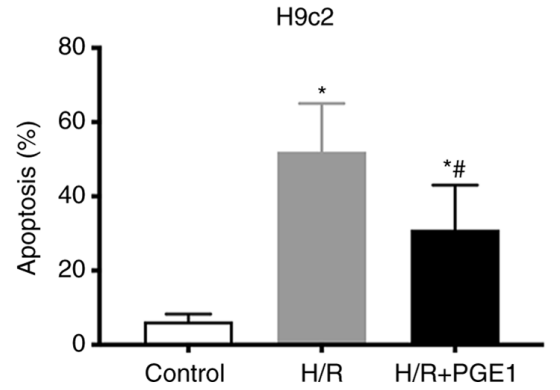

Figure 4. Apoptosis assay with TUNEL staining. (A) Apoptosis analysis of rat myocardium using light microscopy. Scale bar, $20 \mu \mathrm{m}$. "P<0.05 vs. Sham group; ${ }^{\#} \mathrm{P}<0.05$ vs. CA group. (B) Apoptosis analysis of $\mathrm{H} 9 \mathrm{c} 2$ cells using fluorescence microscopy. Scale bar, $50 \mu \mathrm{m},{ }^{*} \mathrm{P}<0.01$ vs. control group; ${ }^{\#} \mathrm{P}<0.01$ vs. H/R group. $\mathrm{CA}$, cardiac arrest; $\mathrm{H} / \mathrm{R}$, hypoxia-reoxygenation; PGE1, prostaglandin E1.

Since mitochondrial disorder plays a vital role in myocardial apoptosis (34,37-39), mPTP opening was assessed. mPTP opening in the inner mitochondrial membrane results in collapse of the membrane potential, matrix swelling and the release of cytochrome $c$ into the cytoplasm, leading to activation of caspase- 3 and induction of apoptosis $(11,37)$. Growing evidence indicates that inhibition of mPTP opening can reduce apoptosis in several pathological conditions $(14,40,41)$. An increase in mPTP was noted following $\mathrm{CA}$ and H/R. However, PGE1 treatment effectively prevented excessive mPTP opening. Similarly, Cour et al (42) reported that cyclosporine A (an inhibitor of mPTP opening) attenuated post-CA syndrome and improved short-term survival in a rabbit model. Moreover, Zhu et al (19) reported that PGE1 pretreatment prevented mPTP opening in a rat model of coronary microembolization. These findings support the hypothesis that PGE1 inhibits mPTP opening.

Cytochrome $c$ and caspase- 3 are downstream proteins in mitochondria-mediated cardiomyocyte apoptosis. When a cell is stimulated by particular pathological factors, macromolecules, including cytochrome $c$, procaspase-2, and procaspase-9, are released through the MPTP. Cytochrome $c$ plays a central role in apoptosis. Once released into the cytosol, it forms a complex known as the apoptosome (43-45). These events allow for the activation of caspase-3, which eventually mediates apoptosis (10-12). To identify the mechanism underlying the antiapoptotic effects of PGE1, the cytosolic protein levels of cytochrome $c$ and cleaved caspase-3 were examined. CA increased cytochrome $c$ and cleaved caspase- 3 expression in the heart, consistent with the findings reported by
Garcia et al (46). PGE1 treatment significantly attenuated the pathological increase in cytochrome $c$ and cleaved caspase-3 that were induced by CA.

The GSK $3 \beta$ pathway is a primary upstream mPTP regulator (47-49). A recent study (50) indicated that a prostaglandin E receptor subtype 4 agonist inhibited $\mathrm{mPTP}$ opening following ischemia-reperfusion in hepatocytes through the GSK3 $\beta$ pathway. To evaluate the mechanism by which PGE1 treatment inhibits mPTP opening, GSK3 $\beta$ activation was measured. GSK3 $\beta$ (Ser9) inhibitory phosphorylation was decreased after CA, whereas PGE1 treatment caused inhibition of (Ser9) phosphorylation of GSK3 $\beta$. Therefore, it may be hypothesized that PGE1 inhibited mPTP opening by inactivating GSK3 $\beta$.

The present study exhibits certain limitations. The CA model used was induced by asphyxia. It remains unknown whether PGE1 also exerts cardioprotective effects in CA induced by ventricular fibrillation. Moreover, primary adult rat cardiac myocytes isolated from the rats would have provided more reliable results than a cardiomyocyte cell line. However, the use $\mathrm{H} 9 \mathrm{c} 2$ cells undergoing $\mathrm{H} / \mathrm{R}$ model remains a reasonable model for the purpose of this study. H9c2 cell line was originally derived from embryonic rat ventricular tissue. Although H9c2 cells are no longer able to beat, they share several characteristics with primary cardiomyocytes (51). The H/R model is also widely used to simulate circulatory arrest $(23,52-54)$ and as a cellular model of cardiac arrest $(23,53,55)$. In addition, inhibitors were not used to further evaluate the role of the GSK $3 \beta$ pathway in the cardioprotective mechanism of PGE1. Lastly, the side effects of PGE1 on hemodynamics were not fully assessed. For example, PGE1 may increase the risk of decreasing blood pressure 
A
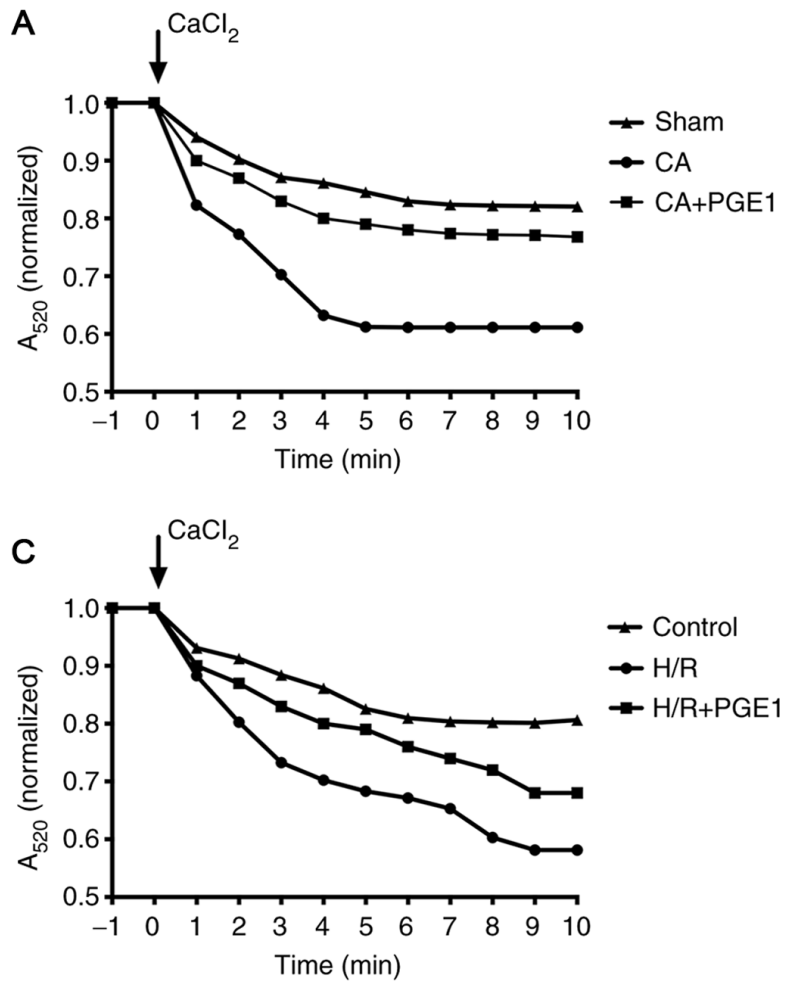

E

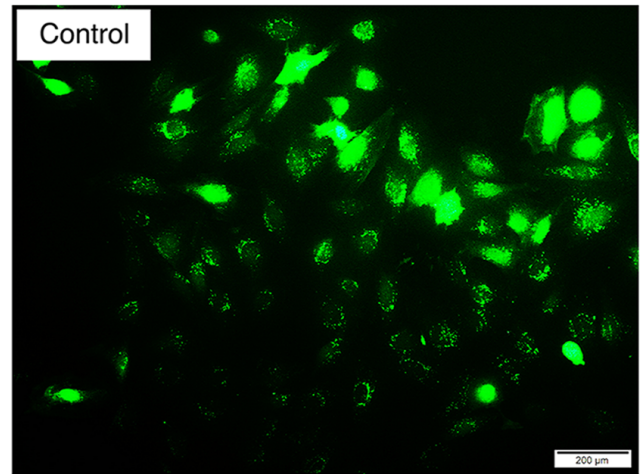

G

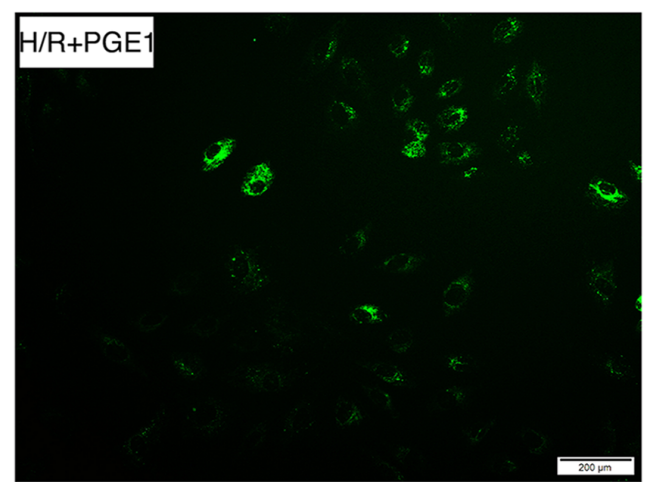

B

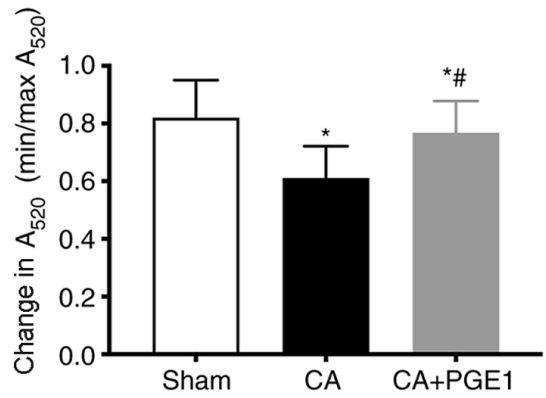

D

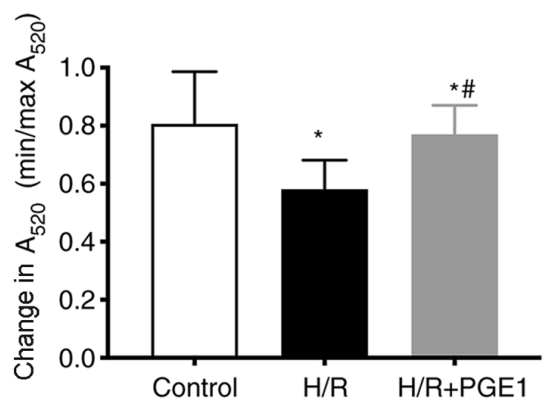

F

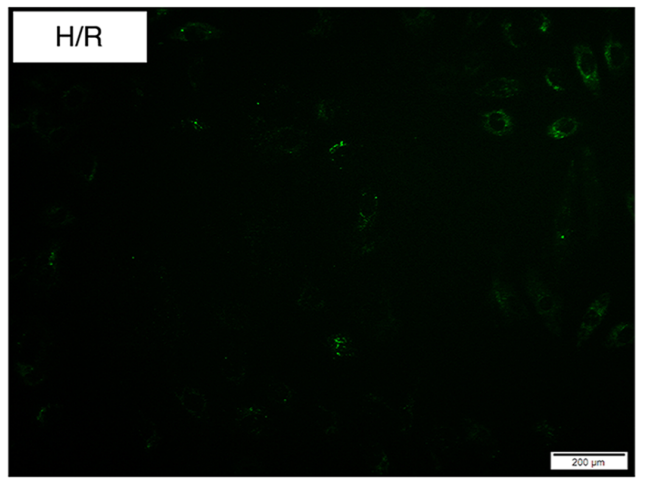

H

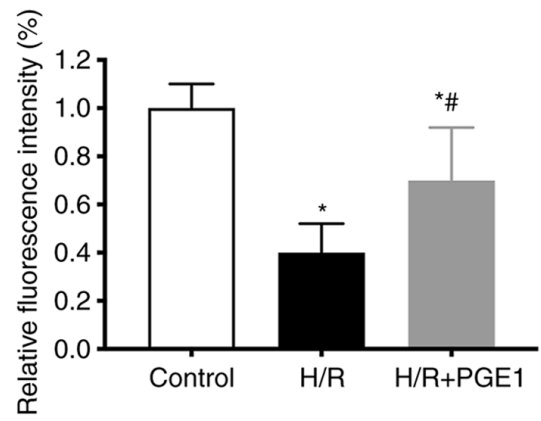

Figure 5. Effects of PGE1 on isolated mPTP opening. (A and B) Downward sloping curves indicating mPTP opening induced by $\mathrm{CaCl}_{2}$ and $\mathrm{A}_{520}$ change $\left(\mathrm{A}_{520}\right.$ at $-1 \mathrm{~min} / \mathrm{A}_{520}$ at $10 \mathrm{~min}$ ) indicating $\mathrm{mPTP}$ opening in rats. ${ }^{*} \mathrm{P}<0.05$ vs. sham group; ${ }^{*} \mathrm{P}<0.05$ vs. CA group. (C and $\left.\mathrm{D}\right)$ Downward sloping curves indicating mPTP opening induced by $\mathrm{CaCl}_{2}$ and $\mathrm{A}_{520}$ change $\left(\mathrm{A}_{520}\right.$ at $-1 \mathrm{~min} / \mathrm{A}_{520}$ at $\left.10 \mathrm{~min}\right)$ indicating $\mathrm{mPTP}$ opening in $\mathrm{H} 9 \mathrm{c} 2$ cells. ${ }^{*} \mathrm{P}<0.05$ vs. control group; ${ }^{\#} \mathrm{P}<0.05 \mathrm{vs}$. $\mathrm{H} / \mathrm{R}$ group. (E-H) The opening of mPTP was assessed by fluorescence intensity of calcein-AM in $\mathrm{H} 9 \mathrm{c} 2$ cells. ${ }^{~} \mathrm{P}<0.01$ vs. control group; $\mathrm{P}<0.01$ vs. $\mathrm{H} / \mathrm{R}$ group. $\mathrm{CA}$, cardiac arrest; H/R, hypoxia-reoxygenation; PGE1, prostaglandin E1; mPTP, mitochondrial permeability transition pore; $\mathrm{A}_{520}$, absorbance at $520 \mathrm{~nm}$.

when used for clinical treatment. The recording of MAP was terminated at $1 \mathrm{~h}$ following ROSC. Although the MAP at $1 \mathrm{~h}$ following ROSC was not significantly different between the CA and CA+PGE1 groups, further studies should be conducted to assess the safety of PGE1 before its cardioprotective effects are investigated in clinical studies. 
A
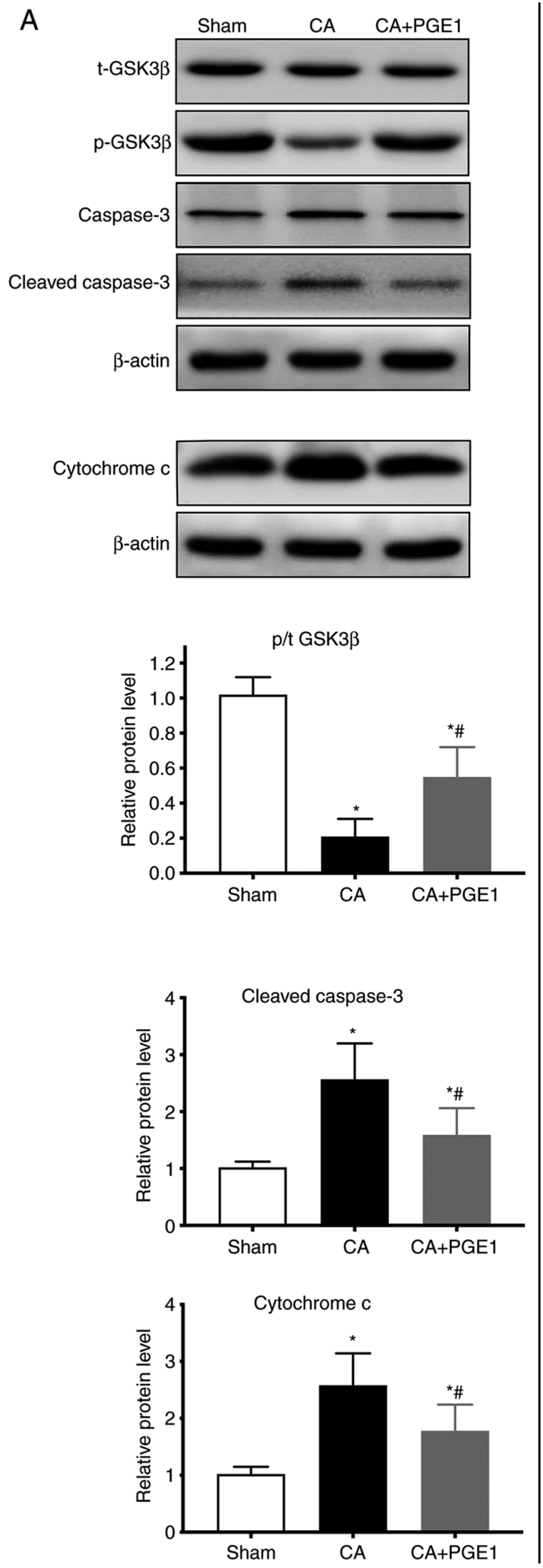

B
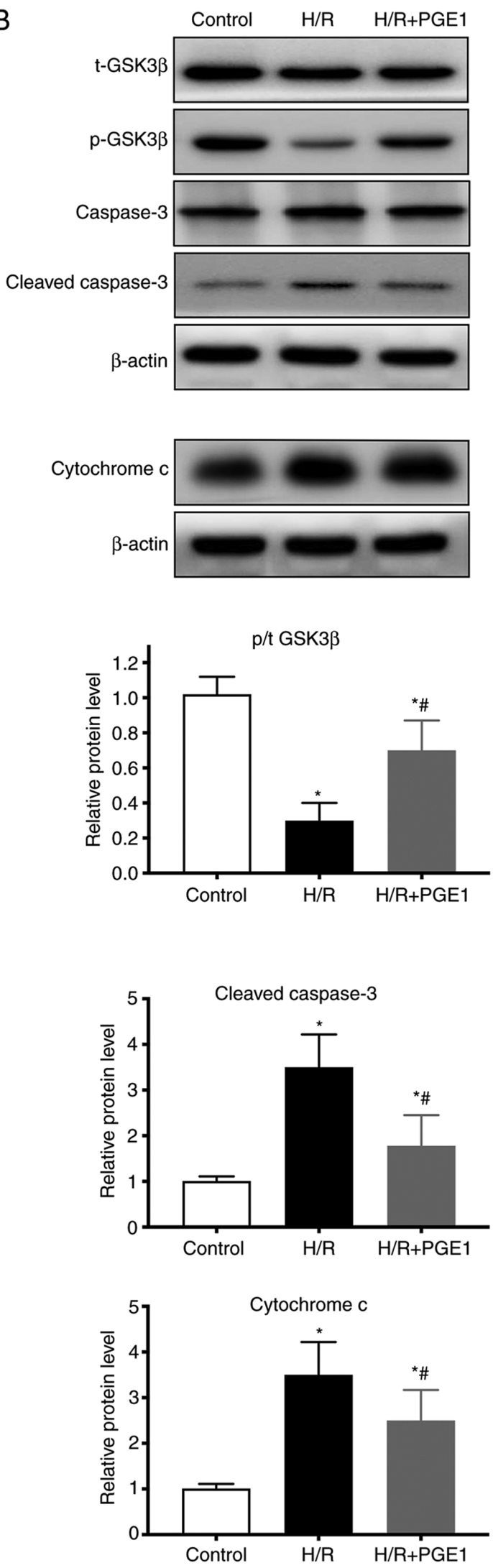

Figure 6. Effects of PGE1 on expression of GSK3 $\beta$, cytochrome $c$ and cleaved caspase-3. (A) p-GSK3 $\beta$, caspase-3 and cytochrome $c$ protein expression in rats, ${ }^{\text {"P }}<0.01$ vs. sham group; ${ }^{\prime} \mathrm{P}<0.01$ vs. CA group. (B) GSK3 $\beta$, cytochrome $c$ and cleaved caspase-3 protein expression in H9c2 cells. "P<0.01 vs. control group; ${ }^{\#} \mathrm{P}<0.01$ vs. H/R group. CA, cardiac arrest; H/R, hypoxia-reoxygenation; PGE1, prostaglandin E1; p, phosphorylated; t, total.

In conclusion, the present study indicated that PGE1 treatment attenuated PAMD at the onset of ROSC and improved the survival rate following CA. Its benefits were partially attributed to inhibition of mitochondria-mediated cardiomyocyte 
apoptosis, which possibly involves the GSK3 $\beta$ pathway. These findings offer significant insight into developing new strategies for the treatment of PAMD.

\section{Acknowledgements}

Not applicable.

\section{Funding}

The present study was supported by the National Key R\&D Program of China (grant no. 2017YFC0908700, 2017YFC0908703), National Natural Science Foundation of China (grant nos. 81772036, 81671952, 81873950, 81873953, 81570401 and 81571934), National S\&T Fundamental Resources Investigation Project (2 grant nos. 018FY100600 and 2018F Y100602), Taishan Pandeng Scholar Program of Shandong Province (grant no. tspd20181220), Taishan Young Scholar Program of Shandong Province (grant nos. tsqn20161065 and tsqn201812129), Key R\&D Program of Shandong Province (grant nos. 2016ZDJS07A14 and 2018GSF118003) and the Fundamental Research Funds of Shandong University (grant no. 2018JC011).

\section{Availability of data and materials}

The datasets used and/or analyzed during the current study are available from the corresponding author on reasonable request.

\section{Authors' contributions}

YC and JW designed the experiments. CS and XF performed the experiments. CS and FX collected and analyzed data. The manuscript was written by CS. FX revised the manuscript critically for important intellectual content. All authors read and approved the manuscript, and agree to be accountable for all aspects of the research in ensuring that the accuracy or integrity of any part of the work are appropriately investigated and resolved. All authors read and approved the final manuscript.

\section{Ethics approval and consent to participate}

The present study was approved by the Animal Use and Care Committee of Shandong University (approval no. KYLL-2018KS-219).

\section{Patient consent for publication}

Not applicable.

\section{Competing interests}

The authors declare that they have no competing interests.

\section{References}

1. Neumar RW: Doubling cardiac arrest survival by 2020: Achieving the American heart association impact goal. Circulation 134: 2037-2039, 2016

2. Andersen LW, Holmberg MJ, Berg KM, Donnino MW and Granfeldt A: In-hospital cardiac arrest: A review. JAMA 321 $1200-1210,2019$.
3. Jentzer JC, Chonde MD, Shafton A, Abu-Daya H, Chalhoub D, Althouse AD and Rittenberger JC: Echocardiographic left ventricular systolic dysfunction early after resuscitation from cardiac arrest does not predict mortality or vasopressor requirements. Resuscitation 106: 58-64, 2016.

4. Laurent I, Monchi M, Chiche JD, Joly LM, Spaulding C, Bourgeois B, Cariou A, Rozenberg A, Carli P, Weber S and Dhainaut JF: Reversible myocardial dysfunction in survivors of out-of-hospital cardiac arrest. J Am Coll Cardiol 40: 2110-2116, 2002.

5. Ruiz-Bailen M, Aguayo de Hoyos E, Ruiz-Navarro S, Díaz-Castellanos MA, Rucabado-Aguilar L, Gómez-Jiménez FJ, Martínez-Escobar S, Moreno RM and Fierro-Rosón J: Reversible myocardial dysfunction after cardiopulmonary resuscitation. Resuscitation 66: 175-181, 2005.

6. Jentzer JC, Chonde MD and Dezfulian C: Myocardial dysfunction and shock after cardiac arrest. Biomed Res Int 2015: 314796, 2015.

7. Chonde M, Flickinger KL, Sundermann ML, Koller AC, Salcido DD, Dezfulian C, Menegazzi JJ and Elmer J: Intra-Arrest administration of cyclosporine and methylprednisolone does not reduce postarrest myocardial dysfunction. Biomed Res Int 2019: 6539050,2019

8. Liu S, Xu J, Gao Y, Shen P, Xia S, Li Z and Zhang M: Multi-organ protection of ulinastatin in traumatic cardiac arrest model. World J Emerg Surg 13: 51, 2018.

9. Heusch G, Boengler K and Schulz R: Inhibition of mitochondrial permeability transition pore opening: The Holy Grail of cardioprotection. Basic Res Cardiol 105: 151-154, 2010

10. Wu HY, Huang CH, Lin YH, Wang CC and Jan TR: Cannabidiol induced apoptosis in human monocytes through mitochondrial permeability transition pore-mediated ROS production. Free Radic Biol Med 124: 311-318, 2018.

11. Sileikyte J and Forte M: The mitochondrial permeability transition in mitochondrial disorders. Oxid Med Cell Longev 2019: 3403075, 2019.

12. Morciano G, Bonora M, Campo G, Aquila G, Rizzo P, Giorgi C, Wieckowski MR and Pinton P: Mechanistic role of mPTP in ischemia-reperfusion injury. Adv Exp Med Biol 982: 169-189, 2017.

13. Huang CH, Tsai MS, Hsu CY, Su YJ, Wang TD, Chang WT and Chen WJ: Post-cardiac arrest myocardial dysfunction is improved with cyclosporine treatment at onset of resuscitation but not in the reperfusion phase. Resuscitation 82 (Suppl 2): S41-S47, 2011.

14. Cour M, Loufouat J, Paillard M, Augeul L, Goudable J, Ovize M and Argaud L: Inhibition of mitochondrial permeability transition to prevent the post-cardiac arrest syndrome: A pre-clinical study. Eur Heart J 32: 226-235, 2011.

15. Hew MR and Gerriets V: Prostaglandin E1. In: StatPearls, Treasure Island, FL, 2019.

16. Weiss T, Fischer D, Hausmann D and Weiss C: Endothelial function in patients with peripheral vascular disease: Influence of prostaglandin E1. Prostaglandins Leukot Essent Fatty Acids 67: 277-281, 2002.

17. Schutte H, Lockinger A, Seeger W and Grimminger F: Aerosolized PGE1, PGI2 and nitroprusside protect against vascular leakage in lung ischaemia-reperfusion. Eur Respir J 18: 15-22, 2001.

18. Johnson RG: Prostaglandin E1 and myocardial reperfusion injury. Crit Care Med 28: 2649-2650, 2000.

19. Zhu H,Ding Y, Xu X, Li M,Fang Y, Gao B, Mao H, Tong G, Zhou L and Huang J: Prostaglandin E1 protects coronary microvascular function via the glycogen synthase kinase $3 \beta$-mitochondrial permeability transition pore pathway in rat hearts subjected to sodium laurate-induced coronary microembolization. Am J Transl Res 9: 2520-2534, 2017.

20. Fang WT, Li HJ and Zhou LS: Protective effects of prostaglandin E1 on human umbilical vein endothelial cell injury induced by hydrogen peroxide. Acta Pharmacol Sin 31: 485-492, 2010.

21. Wei L, Zhao W, Hu Y, Wang X, Liu X, Zhang P and Han F: Exploration of the optimal dose of HOE-642 for the protection of neuronal mitochondrial function after cardiac arrest in rats. Biomed Pharmacother 110: 818-824, 2019.

22. Kim T, Paine MG, Meng H, Xiaodan R, Cohen J, Jinka T, Zheng H, Cranford JA and Neumar RW: Combined intra- and post-cardiac arrest hypothermic-targeted temperature management in a rat model of asphyxial cardiac arrest improves survival and neurologic outcome compared to either strategy alone. Resuscitation 107: 94-101, 2016.

23. Huang CH, Tsai MS, Chiang CY, Su YJ, Wang TD, Chang WT, Chen HW and Chen WJ: Activation of mitochondrial STAT-3 and reduced mitochondria damage during hypothermia treatment for post-cardiac arrest myocardial dysfunction. Basic Res Cardiol 110: 59, 2015 
24. Yin L, Yang Z, Yu H, Qian J, Zhao S, Wang J, Wu X, Cahoon J and Tang W: Changes in sublingual microcirculation is closely related with that of bulbar conjunctival microcirculation in a rat model of cardiac arrest. Shock 45: 428-433, 2016.

25. Keilhoff G, Esser T, Titze M, Ebmeyer U and Schild L: High-potential defense mechanisms of neocortex in a rat model of transient asphyxia induced cardiac arrest. Brain Res 1674: 42-54, 2017.

26. Uray T, Empey PE, Drabek T, Stezoski JP, Janesko-Feldman K, Jackson T, Garman RH, Kim F, Kochanek PM and Dezfulian C: Nitrite pharmacokinetics, safety and efficacy after experimental ventricular fibrillation cardiac arrest. Nitric Oxide 93: 71-77, 2019.

27. Incagnoli P, Ramond A, Joyeux-Faure M, Pepin JL, Levy $P$ and Ribuot C: Erythropoietin improved initial resuscitation and increased survival after cardiac arrest in rats. Resuscitation 80 696-700, 2009

28. McAdams RM, McPherson RJ, Dabestani NM, Gleason CA and Juul SE: Left ventricular hypertrophy is prevalent in sprague-dawley rats. Comp Med 60: 357-363, 2010.

29. Magnet IAM, Ettl F, Schober A, Warenits AM, Grassmann D, Wagne M, Schriefl C, Clodi C, Teubenbacher U, Högler S, et al: Extracorporeal life support increases survival after prolonged ventricular fibrillation cardiac arrest in the rat. Shock 48: 674-680, 2017.

30. Huang X, Zuo L, Lv Y, Chen C, Yang Y, Xin H, Li Y and Qian Y: Asiatic acid attenuates myocardial Ischemia/Reperfusion injury via Akt/GSK-3 $\beta / \mathrm{HIF}-1 \alpha$ signaling in rat $\mathrm{H} 9 \mathrm{c} 2$ Cardiomyocytes. Molecules 21: 1248, 2016.

31. Pu Y, Wu D, Lu X and Yang L: Effects of GCN2/eIF2 $\alpha$ on myocardial ischemia/hypoxia reperfusion and myocardial cells injury. Am J Transl Res 11: 5586-5598, 2019.

32. Kang Y: Management of post-cardiac arrest syndrome. Acute Crit Care 34: 173-178, 2019.

33. Vognsen M,Fabian-Jessing BK, Secher N,Løfgren B,Dezfulian C, Andersen LW and Granfeldt A: Contemporary animal models of cardiac arrest: A systematic review. Resuscitation 113: 115-123, 2017.

34. Piao L, Fang YH, Hamanaka RB, Mutlu GM, Dezfulian C, Archer SL and Sharp WW: Suppression of superoxide-hydrogen peroxide production at Site IQ of mitochondrial Complex I attenuates myocardial stunning and improves postcardiac arrest outcomes. Crit Care Med 48: e133-e140, 2020.

35. Zhao Z, Qu F, Liu R and Xia Y: Differential expression of miR-142-3p protects cardiomyocytes from myocardial ischemia-reperfusion via TLR4/NFkB axis. J Cell Biochem: Nov 20, 2019 (Epub ahead of print). doi: 10.1002/jcb.29506.

36. Gu W, Li C, Yin W, Guo Z, Hou X and Zhang D: Shen-fu injection reduces postresuscitation myocardial dysfunction in a porcine model of cardiac arrest by modulating apoptosis Shock 38: 301-306, 2012.

37. Kuznetsov AV, Javadov S, Margreiter R, Grimm M, Hagenbuchner J and Ausserlechner MJ: The role of mitochondria in the mechanisms of Cardiac Ischemia-Reperfusion injury. Antioxidants (Basel) 8: 454, 2019

38. Zhou X, Qu Y, Gan G, Zhu S, Huang Y, Liu Y, Zhu J, Xie B and Tan Z: Cyclosporine a plus ischemic postconditioning improves neurological function in rats after cardiac resuscitation. Neurocrit Care 32: 812-821, 2020

39. Zheng JH, Xie L, Li N, Fu ZY, Tan XF, Tao R, Qin T and Chen MH: PD98059 protects the brain against mitochondrialmediated apoptosis and autophagy in a cardiac arrest rat model. Life Sci 232: 116618, 2019.

40. Wang H, Chen S, Zhang Y, Xu H and Sun H: Electroacupuncture ameliorates neuronal injury by Pink1/Parkin-mediated mitophagy clearance in cerebral ischemia-reperfusion. Nitric Oxide 91: 23-34, 2019.
41. Sun T, Ding W, Xu T, Ao X, Yu T, Li M, Liu Y, Zhang X, Hou L and Wang J: Parkin regulates programmed necrosis and myocardial ischemia/reperfusion injury by targeting cyclophilin-D. Antioxid Redox Signal 31: 1177-1193, 2019.

42. Cour M, Abrial M, Jahandiez V, Loufouat J, Belaïdi E, Gharib A, Varennes A, Monneret G, Thibault H, Ovize M and Argaud L: Ubiquitous protective effects of cyclosporine A in preventing cardiac arrest-induced multiple organ failure. J Appl Physiol (1985) 117: 930-936, 2014.

43. Chalkias A, Kuzovlev A, Noto A, d'Aloja E and Xanthos T: Identifying the role of cytochrome $\mathrm{c}$ in post-resuscitation pathophysiology. Am J Emerg Med 33: 1826-1830, 2015.

44. Sun L, Jia H, Ma L, Yu M, Yang Y, Liu Y, Zhang H and Zou Z: Metabolic profiling of hypoxia/reoxygenation injury in $\mathrm{H} 9 \mathrm{c} 2$ cells reveals the accumulation of phytosphingosine and the vital role of Dan-Shen in Xin-Ke-Shu. Phytomedicine 49: 83-94, 2018.

45. Borutaite V and Brown GC: Mitochondria in apoptosis of ischemic heart. FEBS Lett 541: 1-5, 2003.

46. Garcia NA, Moncayo-Arlandi J, Vazquez A, Genovés P, Calvo CJ, Millet J, Martí N, Aguado C, Knecht E, Valiente-Alandi I, et al: Hydrogen sulfide improves cardiomyocyte function in a cardiac arrest model. Ann Transplant 22: 285-295, 2017.

47. Yang K, Chen Z, Gao J, Shi W, Li L, Jiang S, Hu H, Liu Z, Xu D and $\mathrm{Wu}$ L: The key roles of GSK-3 $\beta$ in regulating mitochondrial activity. Cell Physiol Biochem 44: 1445-1459, 2017.

48. Nikolaou PE, Boengler K, Efentakis P, Vouvogiannopoulou K, Zoga A, Gaboriaud-Kolar N, Myrianthopoulos V, Alexakos P, Kostomitsopoulos N, Rerras I, et al: Investigating and re-evaluating the role of glycogen synthase kinase 3 beta kinase as a molecular target for cardioprotection by using novel pharmacological inhibitors. Cardiovasc Res 115: 1228-1243, 2019.

49. Tanaka T, Saotome M, Katoh H, Satoh T, Hasan P, Ohtani H, Satoh H, Hayashi $\mathrm{H}$ and Maekawa Y: Glycogen synthase kinase- $3 \beta$ opens mitochondrial permeability transition pore through mitochondrial hexokinase II dissociation. J Physiol Sci 68: 865-871, 2018.

50. Cai LL, Xu HT, Wang QL, Zhang YQ, Chen W, Zheng DY, Liu F, Yuan HB, Li YH and Fu HL: EP4 activation ameliorates liver ischemia/reperfusion injury via ERK1/2GSK3 $\beta$-dependent MPTP inhibition. Int J Mol Med 45: 1825-1837, 2020.

51. Kimes BW and Brandt BL: Properties of a clonal muscle cell line from rat heart. Exp Cell Res 98: 367-381, 1976.

52. Kwon WY, Suh GJ, Kim KS, Jung YS, Kim SH, Lee AR, You KM and Park MJ: Niacin and selenium attenuate brain injury after cardiac arrest in rats by up-regulating DJ-1-akt signaling. Crit Care Med 46: e788-e796, 2018.

53. Zhang R, Liu B, Fan X, Wang W, Xu T, Wei S, Zheng W, Yuan Q, Gao L, Yin X, et al: Aldehyde dehydrogenase 2 protects against post-cardiac arrest myocardial dysfunction through a novel mechanism of suppressing mitochondrial reactive oxygen species production. Front Pharmacol 11: 373, 2020.

54. Zhou T, Lin H, Jiang L, Yu T, Zeng C, Liu J and Yang Z: Mild hypothermia protects hippocampal neurons from oxygen-glucose deprivation injury through inhibiting caspase-3 activation. Cryobiology 80: 55-61, 2018

55. Yeh $\mathrm{CH}$, Chen TP, Wang YC, Lin YM and Fang SW: MicroRNA-27a regulates cardiomyocytic apoptosis during cardioplegia-induced cardiac arrest by targeting interleukin 10-related pathways. Shock 38: 607-614, 2012.

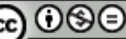

This work is licensed under a Creative Common Attribution-NonCommercial-NoDerivatives 4.0 International (CC BY-NC-ND 4.0) License. 